Nonlinear Analysis and Differential Equations, Vol. 4, 2016, no. 10, 467 - 476 HIKARI Ltd, www.m-hikari.com

http://dx.doi.org/10.12988/nade.2016.6747

\title{
The k- $\eta$-Hyperbolic Functions
}

\author{
Rubén A. Cerutti \\ Departamento de Matemática, Facultad de Ciencias Exactas \\ Universidad Nacional del Nordeste \\ Avda. Libertad 5540, (3400) Corrientes, Argentina
}

Copyright (c) 2016 Rubén A. Cerutti. This article is distributed under the Creative Commons Attribution License, which permits unrestricted use, distribution, and reproduction in any medium, provided the original work is properly cited.

\begin{abstract}
The main objetive of this paper is to present and discuss a family of functions dependent on five parameters that generalize the one introduced by A. Ungar [8]. The action of Riemann-Liouville fractional operators over them is evaluated as well their Laplace transform. Particular case are shown.
\end{abstract}

Mathematics Subject Classification: 26A33; 33E12

Keywords: k-Gamma function. k-Mittag-Leffler function. Fractional operators

\section{Introduction and preliminary notes}

By using the k-Gamma function and the Pochhammer k-symbol [2] we define a family of functions that are analogous to the $\eta$-Hyperbolic functions due to A. Ungar ([8]). As special cases, the new functions contains the classical trigonometric functions sine and cosine, also the hyperbolic functions sinh and cosh as well its fractional versions. Besides that, for certain values of the variable, the k-Mittag-Leffler function is obtained.

We begin remembering basic notions that will need for further development of this paper.

Definition 1 ([1]) Let $x \in \mathbb{C}, k \in \mathbb{R}$ and $n \in \mathbb{N}^{+}$. The Pochhammer $k$-symbol is given by

$$
(x)_{n, k}=x \cdot(x+k) \cdot(x+2 k) \ldots(x+(n-1) k) .
$$


Definition 2 ([1]) Let $z \in \mathbb{C}, \operatorname{Re}(z)>0$. The $k$-Gamma function is

$$
\Gamma_{k}(z)=\int_{0}^{\infty} t^{z-1} e^{-\frac{t^{k}}{k}} d t
$$

Among the many properties that link the Pochhammer k-symbol and the k-Gamma function, we will highlight those specified in the following

Proposition 1 Given $z \in \mathbb{C}-k \mathbb{Z}^{-} ; k, s>0$ and $n \in \mathbb{N}^{+}$, the following identity holds

1.

$$
(z)_{n, s}=\left(\frac{s}{k}\right)^{n}\left(\frac{k z}{s}\right)_{n, k}
$$

2.

$$
\Gamma_{s}(z)=\left(\frac{s}{k}\right)^{\frac{z}{k}-1} \Gamma_{k}\left(\frac{k z}{s}\right)
$$

For the proof we remit to Proposition 4 in [8].

The well know Mittag-Leffler function defined by the following series:

$$
\begin{gathered}
E_{\alpha}(z)=\sum_{n=0}^{\infty} \frac{z^{n}}{\Gamma(\alpha n+1)}, \quad \operatorname{Re}(\alpha)>0 . \\
E_{\alpha, \beta}(z)=\sum_{n=0}^{\infty} \frac{z^{n}}{\Gamma(\alpha n+\beta)}, \quad \beta \in \mathbb{C}, \operatorname{Re}(\alpha)>0 .
\end{gathered}
$$

has been subject to several generalizations among which we highlight the one introduced by us in [2]

$$
E_{k, \alpha, \beta}^{\gamma}(z)=\sum_{n=0}^{\infty} \frac{(\gamma)_{n, k}}{\Gamma_{k}(\alpha n+\beta) n !} z^{n}
$$

where $(\gamma)_{n, k}$ is the Pochhammer k-symbol given by (I.1), and $\Gamma_{k}(\alpha n+\beta)$ is the k-Gamma function (I.2)

Definition 3 The Riemann-Liouville integral of order $\nu$ of a function $f$ is given by

$$
I^{\nu} f(t)=\frac{1}{\Gamma(\nu)} \int_{0}^{t}(t-\tau)^{\nu-1} f(\tau) d \tau ; \quad t \in \mathbb{R}^{+}, \nu \in \mathbb{C}, \operatorname{Re}(\nu)>0 .
$$

Definition 4 The Riemann-Liouville fractional derivative of order $\nu$ of a function $f$ is given by

$$
D^{\nu} f(t)=\left(\frac{d}{d t}\right)^{n} I^{n-\nu} f(t), \quad t>0, n=[\operatorname{Re}(\nu)]+1 .
$$


Definition 5 Let $f: \mathbb{R}^{+} \rightarrow \mathbb{R}$ be an exponential order function and piecewise continuous. The Laplace transform of $f$ is

$$
\mathcal{L}\{f(t)\}(s)=\int_{0}^{\infty} e^{-s t} f(t) d t
$$

provided that the integral in (I.10) exists.

The Laplace transform of the fractional Riemann-Liouville operator are given by the following:

Proposition 2 Let $\alpha \in \mathbb{C}, \operatorname{Re}(\alpha)>0$, then

$$
\mathcal{L}\left\{I^{\alpha} f(t)\right\}(s)=\frac{\mathcal{L}\{f(t)\}(s)}{s^{\alpha}}
$$

and if $\alpha$ is such that verifies $m-1<\operatorname{Re}(\alpha) \leq m$, then

$$
\mathcal{L}\left\{D^{\alpha} f(t)\right\}(s)=s^{\alpha} \mathcal{L}\{f(t)\}(s)-\sum_{j=0}^{m-1} s^{j} D^{\alpha-j-1} f(0) .
$$

\section{Main results}

In his paper entitled Higher order $\alpha$-Hyperbolic functions [8] A. Ungar introduced the $\alpha$-Hyperbolic functions by the expression

$$
F_{n, r}^{\alpha}(z)=\sum_{k=0}^{\infty} \frac{\alpha^{k}}{(n k+r) !} z^{n k+r}
$$

for any pair of integer $(n, r), n \geq 2 ; 0 \leq r<n$, and any complex constant $\alpha$.

By using the k-gamma functions and the Pochhammer k-symbol we define the $\mathrm{k}-\eta$-Hyperbolic function by

$$
{ }_{k} F_{\alpha, \beta+k}^{\gamma, \eta}(z)=\sum_{n=0}^{\infty} \frac{(\gamma)_{n, k} \eta^{n}}{\Gamma_{k}(\alpha n+\beta+k) n !} z^{\frac{\alpha}{k} n+\frac{\beta}{k}}
$$

for $\alpha, \gamma, k$ real positive number and $\beta \geq 0, \eta \in \mathbb{C}$.

Easily, we can see that for $\gamma=k=1$, and, $\alpha$ and $\beta$ integers, $\alpha \geq 2$, $0 \leq \beta<\alpha$, we have (II.1).

Moreover, also may be the following special cases:

1. If $\eta=0$

$$
{ }_{k} F_{\alpha, \beta+k}^{\gamma, 0}(z)=\frac{z^{\frac{\beta}{k}}}{\Gamma_{k}(\beta+k)}
$$


2. If $\eta=0, k=1$ and $\beta \in \mathbb{N}$

$$
{ }_{1} F_{\alpha, \beta+k}^{\gamma, 0}(z)=\frac{z^{\beta}}{\beta !}
$$

which coincides with the 0-hyperbolic function given in the formulae (2) of $[8]$.

3. If $\eta=1$,

$$
\begin{aligned}
{ }_{k} F_{\alpha, \beta+k}^{\gamma, 1}\left(z^{k}\right) & =z^{\frac{\beta}{k}} \sum_{n=0}^{\infty} \frac{(\gamma)_{n, k}}{\Gamma_{k}(\alpha n+\beta+k) n !} z^{\frac{\alpha}{k} n} \\
& =z^{\frac{\beta}{k}} E_{k, \alpha, \beta+k}^{\gamma}\left(z^{\frac{\alpha}{k}}\right)
\end{aligned}
$$

where $E_{k, \alpha, \beta+k}^{\gamma}\left(z^{\frac{\alpha}{k}}\right)$ is the k-Mittag-Leffler function given in (I.7).

4. If $\beta=\alpha-k, \beta>0$

$$
{ }_{k} F_{\alpha, \alpha-k+k}^{\gamma, \eta}(z)=z^{\frac{\alpha}{k}-1} \sum_{n=0}^{\infty} \frac{(\gamma)_{n, k}}{\Gamma_{k}(\alpha(n+1)) n !}\left(\eta z^{\frac{\alpha}{k}}\right)^{n},
$$

then

$$
{ }_{k} F_{\alpha, \alpha-k+k}^{\gamma, \eta}(z)=z^{\frac{\alpha}{k}-1} E_{k, \alpha, \alpha}^{\gamma}\left(\eta z^{\frac{\alpha}{k}}\right)={ }_{k} e_{\gamma, \alpha,}^{\eta z}
$$

where ${ }_{k} e_{\gamma, \alpha}^{z}$ denotes the k- $\alpha$-Exponential function defined in [4].

Then

$$
{ }_{k} F_{\alpha,(\alpha-k)+k}^{\gamma, \eta}(z)={ }_{k} e_{\gamma, \alpha}^{\eta z}
$$

5. Considering the function ${ }_{k} F_{\alpha, \beta+k}^{\gamma, \eta}(z)$ and grouping according to the parity of the summation index, we have

$$
\begin{aligned}
{ }_{k} F_{\alpha, \beta+k}^{\gamma, \eta}(z) & =\sum_{n=0}^{\infty} \frac{(\gamma)_{n, k} \eta^{n}}{\Gamma_{k}(\alpha n+\beta+k) n !} z^{\frac{\alpha}{k} n+\frac{\beta}{k}} \\
& =z^{\frac{\beta}{k}}\left\{\sum_{n=0}^{\infty} \frac{(\gamma)_{2 n, k} \eta^{2 n} z^{\frac{\alpha}{k} 2 n}}{\Gamma_{k}(\alpha 2 n+\beta+k)(2 n) !}\right. \\
& \left.+\sum_{n=0}^{\infty} \frac{(\gamma)_{2 n+1, k} \eta^{2 n+1} z^{\frac{\alpha}{k}(2 n+1)}}{\Gamma_{k}(\alpha(2 n+1)+\beta+k)(2 n+1) !}\right\}
\end{aligned}
$$

Then we may write

$$
{ }_{k} F_{\alpha, \beta+k}^{\gamma, \eta}(z)=z^{\frac{\beta}{k}}\left\{{ }_{k} C_{\alpha, \beta+k}^{\gamma, \eta}(z)+{ }_{k} S_{\alpha, \beta+k}^{\gamma, \eta}(z)\right\}
$$


where

$$
{ }_{k} C_{\alpha, \beta+k}^{\gamma, \eta}(z)=\sum_{n=0}^{\infty} \frac{(\gamma)_{2 n, k} \eta^{2 n} z^{\frac{\alpha}{k} 2 n}}{\Gamma_{k}(\alpha 2 n+\beta+k)(2 n) !}
$$

and

$$
{ }_{k} S_{\alpha, \beta+k}^{\gamma, \eta}(z)=\sum_{n=0}^{\infty} \frac{(\gamma)_{2 n+1, k} \eta^{2 n+1} z^{\frac{\alpha}{k}(2 n+1)}}{\Gamma_{k}(\alpha(2 n+1)+\beta+k)(2 n+1) !}
$$

Choosing the particular value $\beta=\alpha-k$, it has

$$
\begin{aligned}
{ }_{k} C_{\alpha, \alpha}^{\gamma, \eta}(z) & =\sum_{n=0}^{\infty} \frac{(\gamma)_{2 n, k} \eta^{2 n} z^{\frac{\alpha}{k} 2 n}}{\Gamma_{k}(\alpha 2 n+\alpha)(2 n) !} \\
& =\sum_{n=0}^{\infty} \frac{(\gamma)_{2 n, k} \eta^{2 n} z^{\frac{\alpha}{k} 2 n}}{\Gamma_{k}(\alpha(2 n+1))(2 n) !}
\end{aligned}
$$

and if $\gamma=1, \eta=1, \alpha=1=k$, one has

$$
{ }_{1} C_{1,1}^{1,1}(z)=\sum_{n=0}^{\infty} \frac{z^{2 n}}{\Gamma(2 n+1)}=\cosh (z)
$$

If $\eta=i \nu, \gamma=1, \nu>0, \alpha=k=1$, from (II.15) we obtain

$$
{ }_{1} C_{1,1}^{1, i \nu}(z)=\sum_{n=0}^{\infty} \frac{(-1)^{n} \nu^{2 n} z^{2 n}}{(2 n) !}=\cos (\nu z)
$$

By (II.15), (II.16) and (II.17) we designates ${ }_{k} C_{\alpha, \beta+k}^{\gamma, \eta}(z)$ the $\eta$-hyperbolic cosine function of order $\alpha$ and $(\beta+k)$-th kind.

Analogously, making in (II.14) the following choice of parameters, we have,

If $\beta=\alpha-k$, then

$$
{ }_{k} S_{\alpha, \alpha}^{\gamma, \eta}(z)=\sum_{n=0}^{\infty} \frac{(\gamma)_{2 n+1, k} \eta^{2 n+1} z^{\frac{\alpha}{k}(2 n+1)}}{\Gamma_{k}(\alpha(2 n+2))(2 n+1) !}
$$

and if $\gamma=\eta=\alpha=k=1$,

$$
{ }_{1} S_{1,1}^{1,1}(z)=\sum_{n=0}^{\infty} \frac{z^{(2 n+1)}}{(2 n+1) !}=\sinh (z)
$$


And, if $\eta=i \nu, \nu>0, \gamma=\alpha=k=1$

$$
{ }_{1} S_{1,1}^{1, i \nu}(z)=i \sum_{n=0}^{\infty} \frac{(-1)^{n} \nu^{2 n+1} z^{2 n+1}}{(2 n+1) !}=i \sin (\nu z)
$$

If in (II.18) we take $\eta=-1$, and $\gamma=\alpha=k=1$, it results

$$
{ }_{k} S_{1,1}^{1,-1}(z)=-\sinh (z)
$$

By (II.19), (II.20) and (II.21), we define ${ }_{k} S_{\alpha, \beta+k}^{\gamma, \eta}(z)$ the $\eta$-hyperbolic sine function of order $\alpha$ and $(\beta+k)$-th kind.

From (II.12), when $\beta=\alpha-k$, we may write

$$
{ }_{k} F_{\alpha, \alpha}^{\gamma, \eta}(z)=z^{\frac{\alpha}{k}-1}\left\{{ }_{k} C_{\alpha, \alpha}^{\gamma, \eta}(z)+{ }_{k} S_{\alpha, a l p h a}^{\gamma, \eta}(z)\right\}
$$

and when $\alpha=k=\gamma=1$

$$
e^{\eta z}={ }_{1} F_{1,1}^{\gamma, \eta}(z)=\cosh (\eta z)+\sinh (\eta z)
$$

Further on we will see other particular cases of the function introduced in (II.2) allowing us to meet with the functions sinh and cosh.

By analogous considerations to those of Theorem 1 by Srivastava and Tomovski [7], it can be shown that the $\mathrm{k}-\eta$-Hyperbolic function is an entire function in the complex plane. Then we have the following

Theorem 1 The $k$ - $\eta$-Hyperbolic function ${ }_{k} F_{\alpha, \beta+k}^{\gamma, \eta}(z)$ defined by (II.2) is an entire function in $\mathbb{C}$.

Lemma 1 Let $E_{k, \alpha, \beta}^{\gamma}\left(\eta z^{\frac{\alpha}{k}}\right)$ be a k-Mittag-Leffler function; then

\section{Lemma 2}

$$
\frac{d}{d z}\left(E_{k, \alpha, \beta}^{\gamma}\left(\eta z^{\frac{\alpha}{k}}\right)\right)=\frac{\alpha}{k}(\gamma)_{1, k} E_{k, \alpha, \alpha+\beta}^{\gamma+k}\left(\eta z^{\frac{\alpha}{k}}\right)
$$

$$
\frac{d}{d z}\left({ }_{k} F_{\alpha, \beta+k}^{\gamma, \eta}(z)\right)=\frac{\beta}{k} z^{-1}{ }_{k} F_{\alpha, \beta+k}^{\gamma, \eta}(z)+\frac{\alpha}{k} \gamma_{k} F_{\alpha, \alpha+\beta+k}^{\gamma+k, \eta}(z)
$$

Proof.

$$
\begin{gathered}
\frac{d}{d z}\left({ }_{k} F_{\alpha, \beta+k}^{\gamma, \eta}(z)\right)=\frac{d}{d z}\left(z^{\frac{\beta}{k}} E_{k, \alpha, \beta+k}^{\gamma}\left(\eta z^{\frac{\alpha}{k}}\right)\right. \\
=\frac{\beta}{k} z^{\frac{\beta}{k}-1} E_{k, \alpha, \beta+k}^{\gamma}\left(\eta z^{\frac{\alpha}{k}}\right)+z^{\frac{\beta}{k}} \frac{d}{d z}\left(E_{k, \alpha, \beta+k}^{\gamma}\left(\eta z^{\frac{\alpha}{k}}\right)\right) \\
=\frac{\beta}{k} z^{-1} z^{\frac{\beta}{k}} E_{k, \alpha, \beta+k}^{\gamma}\left(\eta z^{\frac{\alpha}{k}}\right)+\frac{\alpha}{k}(\gamma)_{1, k} z^{\frac{\beta}{k}} E_{k, \alpha, \alpha+\beta+k}^{\gamma+k, \eta}\left(z^{\frac{\alpha}{k}}\right) \\
=\frac{\beta}{k} z^{-1}{ }_{k} F_{\alpha, \beta+k}^{\gamma, \eta}(z)+\frac{\alpha}{k}(\gamma)_{1, k} F_{\alpha, \alpha+\beta+k}^{\gamma+k, \eta}(z)
\end{gathered}
$$




\section{II.1 Laplace Transform}

We start rewriting ${ }_{k} F_{k, \alpha, \beta+k}^{\gamma, \eta}(z)$ in term of a three parameters Mittag-Leffler function.

As we know, from the Definition (II.2) we have

$$
{ }_{k} F_{\alpha, \beta+k}^{\gamma, \eta}(z)=\sum_{n=0}^{\infty} \frac{(\gamma)_{n, k} \eta^{n}}{\Gamma_{k}(\alpha n+\beta+k) n !} z^{\frac{\alpha}{k} n+\frac{\beta}{k}} .
$$

From the (I.3) and (I.4) can be written

$$
(\gamma)_{n, k}=k^{n}\left(\frac{\gamma}{k}\right)_{n}
$$

and

$$
\Gamma_{k}(\gamma)=k^{\frac{\gamma}{k}-1} \Gamma\left(\frac{\gamma}{k}\right)
$$

Then

$$
\begin{aligned}
{ }_{k} F_{\alpha, \beta+k}^{\gamma, \eta}(z) & =\sum_{n=0}^{\infty} \frac{k^{n}\left(\frac{\gamma}{k}\right)_{n} \eta^{n}}{k^{\frac{\alpha n+\beta}{k}} \Gamma\left(\frac{\alpha n+\beta}{k}+1\right) n !} z^{\frac{\alpha}{k} n+\frac{\beta}{k}} \\
& =k^{-\frac{\beta}{k}} z^{\frac{\beta}{k}} \sum_{n=0}^{\infty} \frac{\left(\frac{\gamma}{k}\right)_{n}\left(k^{-\frac{\alpha}{k}} \eta z^{\frac{\alpha}{k}}\right)^{n}}{\Gamma\left(\frac{\alpha}{k} n+\frac{\beta}{k}+1\right) n !}
\end{aligned}
$$

i.e.

$$
{ }_{k} F_{\alpha, \beta+k}^{\gamma, \eta}(z)=\left(\frac{z}{k}\right)^{\frac{\beta}{k}}{ }_{k} E_{\frac{\alpha}{k}, \frac{\beta}{k}+1}^{\frac{\gamma}{k}}\left(\eta\left(\frac{z}{k}\right)^{\frac{\alpha}{k}}\right)
$$

Lemma 3 The Laplace transform of ${ }_{k} F_{\alpha, \beta+k}^{\gamma, \eta}(z)$ is

$$
\mathcal{L}\left\{{ }_{k} F_{\alpha, \beta+k}^{\gamma, \eta}(z)\right\}(s)=\frac{s^{-1}}{(k s)^{\frac{\beta}{k}}} \frac{1}{\left(1-\eta\left(\frac{1}{k s}\right)^{\frac{\alpha}{k}}\right)^{\frac{\gamma}{k}}}
$$

Proof.

Taking into account formulae (11.8) from [3] and making appropiate substitution we have (II.31).

\section{II.1.1 Particular cases.}

1. If $\alpha=\gamma=\eta=k=1$, and $\beta=0$

$$
\mathcal{L}\left\{{ }_{1} F_{1,1}^{1,1}(z)\right\}(s)=\frac{1}{s} \frac{1}{\left(1-\frac{1}{s}\right)}=\frac{1}{s-1} ;
$$


which is in accordance with that

$$
{ }_{1} F_{1,1}^{1,1}(z)=\sum_{n=0}^{\infty} \frac{z^{n}}{\Gamma(n+1)}=e^{z}
$$

and

$$
\mathcal{L}\left\{e^{z}\right\}(s)=\frac{1}{s-1}
$$

2. If $\alpha=2, \beta=1 ; \gamma=\eta=k=1$

$$
\mathcal{L}\left\{{ }_{1} F_{2,2}^{1,1}(z)\right\}(s)=\frac{1}{s^{2}} \frac{1}{\left(1-\frac{1}{s^{2}}\right)}=\frac{1}{s^{2}-1} ;
$$

and we know that

$$
{ }_{1} F_{2,2}^{1,1}(z)=\sum_{n=0}^{\infty} \frac{z^{2 n+1}}{\Gamma(2 n+2)}=\sinh (z)
$$

3. If $\alpha=2, \beta=0, \gamma=\eta=k=1$

$$
\mathcal{L}\left\{{ }_{1} F_{2,1}^{1,1}(z)\right\}(s)=\mathcal{L}\{\cosh (z)\}(s)=\frac{s}{s^{2}-1}
$$

4. If $\alpha=2, \beta=0, \gamma=k=1, \eta=-1$

$$
\mathcal{L}\left\{z^{-1}{ }_{1} F_{2,1}^{1,-1}(z)\right\}(s)=\mathcal{L}\{\cos (z)\}(s)=\frac{s}{s^{2}+1}
$$

5. If $\alpha=2, \beta=1 \gamma=k=1$, and $\eta=-1$

$$
\mathcal{L}\left\{{ }_{1} F_{2,2}^{1,-1}(z)\right\}(s)=\mathcal{L}\{\sin (z)\}(s)=\frac{1}{s^{2}+1}
$$

In the next we will show some properties of the k- $\eta$-Hyperbolic function associated with Riemann-Liouville fractional operators.

Theorem 2 Let $I^{\mu}$ be the Riemann-Liouville fractional integral operator (I.8). Then holds

$$
I^{\mu}\left({ }_{k} F_{\alpha, \beta+k}^{\gamma, \eta}(z)\right)(t)=\left(\frac{t}{k}\right)^{\mu}{ }_{k} F_{\alpha, \beta+k+\mu k}^{\gamma, \eta}(t)
$$

Proof.

Taking into account (II.30), applying Theorem 11.3, formulae (11.11) from [3], it results

$$
\begin{aligned}
I^{\mu}\left[{ }_{k} F_{\alpha, \beta+k}^{\gamma, \eta}(z)\right](t) & =\left(\frac{t}{k}\right)^{\frac{\beta}{k}+\mu} E_{\frac{\alpha}{k}, \frac{\beta}{k}+1+\mu}^{\frac{\gamma}{k}}\left(\eta t^{\frac{\alpha}{k}}\right) \\
& =\left(\frac{t}{k}\right)^{\mu}{ }_{k} F_{\alpha, \beta+k+\mu k}^{\gamma, \eta}(t)
\end{aligned}
$$


Theorem 3 Let $D^{\mu}$ be the Riemann-Liouville fractional derivative definied by (I.9), then holds

$$
D^{\mu}\left({ }_{k} F_{\alpha, \beta+k}^{\gamma, \eta}(z)\right)(t)=\left(\frac{t}{k}\right)^{-\mu}{ }_{k} F_{\alpha, \beta+k-\mu k}^{\gamma, \eta}(t)
$$

Proof.

Analogously to what was done in the previous theorem and applying Theorem 11.3, formula (11.13) from [3] we have

$$
\begin{aligned}
D^{\mu}\left[{ }_{k} F_{\alpha, \beta+k}^{\gamma, \eta}(z)\right](t) & =\left(\frac{t}{k}\right)^{\frac{\beta}{k}-\mu} E_{\frac{\alpha}{k}, \frac{\beta}{k}+1-\mu}^{\frac{\gamma}{k}}\left(\eta t^{\frac{\alpha}{k}}\right) \\
& =\left(\frac{t}{k}\right)^{-\mu}{ }_{k} F_{\alpha, \beta+k-\mu k}^{\gamma, \eta}(t)
\end{aligned}
$$

Example: If $\alpha=2, \beta=1 \gamma=k=1, \quad \eta=-1$, and $\mu=\frac{\alpha}{k}=2$ we have

$$
\begin{aligned}
D^{2}\left[{ }_{1} F_{2,2}^{1,-1}(z)\right](t) & =D^{2}\left[\sum_{n=0}^{\infty} \frac{(-1)^{n} z^{2 n+1}}{\Gamma(2 n+2)}\right](t) \\
& =D^{2}[\sin (z)](t) \\
& =t^{-2}{ }_{1} F_{2,0}^{1,-1}(t)
\end{aligned}
$$

By other hand, from (II.30) we have

$$
\begin{aligned}
t^{-2}{ }_{1} F_{2,0}^{1,-1}(t) & =t^{1-2} E_{2,1+1-2}^{1}\left(-t^{2}\right) \\
& =t^{-1} \sum_{n=0}^{\infty} \frac{(-1)^{n} t^{2 n}}{\Gamma(2 n-1+1)} \\
& =-\sum_{n=0}^{\infty} \frac{(-1)^{n} t^{2 n+1}}{(2 n+1) !} \\
& =-\sin t
\end{aligned}
$$

\section{References}

[1] R. Díaz and E. Pariguan, On hypergeometric functions and $\mathrm{k}$ Pochhammer symbol, Divulgaciones Matemáticas, 15 (2007), no. 2, 179192. 
[2] G. A. Dorrego, R. A. Cerutti, The k-Mittag-Leffler function, Int. J. Contemp. Math. Sciences, 7 (2012), no. 15, 705-716.

[3] H. J. Haubold, A. M. Mathai and R. K. Saxena, Mittag-Leffler Functions and Their Applications, Journal of Applied Mathematics, 2011 (2011), Article ID 298628, 1-51. http://dx.doi.org/10.1155/2011/298628

[4] L. L. Luque, R. A. Cerutti, The k- $\alpha$ Exponential Function, Int. Journal of Math. Analysis, 7 (2013), no. 11, 535-542.

[5] S. Mubeen, G. M. Habibullah, k-Fractional Integrals and Application, Int. J. Contemp. Math. Science, 7 (2012), no. 2, 89-94.

[6] S. Nagamani, Generalized fractional hyperbolic functions, Bulletin of the Marathwada Math. Society, 15 (2014), no. 1, 54-61.

[7] H. M. Srivastava and Z. Tomovski, Fractional calculus with an integral operator containing a generalized Mittag-Leffler function in the kernel, Appl. Math. Comput., 211 (2009), 198-210. http://dx.doi.org/10.1016/j.amc.2009.01.055

[8] A. Ungar, Higher order $\alpha$-Hyperbolic functions, Indian J. Pure and Applied Math., 15 (1984), no. 3, 301-304.

Received: July 21, 2016; Published: September 1, 2016 